\title{
Regularity of Node Distribution Impacts Conduction Velocities in Finite Element Simulations of the Heart
}

\author{
Eike M Wülfers ${ }^{1,2}$, Olaf Dössel ${ }^{3}$, Gunnar Seemann $^{1,2}$ \\ ${ }^{1}$ Institute for Experimental Cardiovascular Medicine, University Heart Center \\ Freiburg · Bad Krozingen and Medical Center, University of Freiburg, Freiburg, Germany \\ ${ }^{2}$ Faculty of Medicine, University of Freiburg, Freiburg, Germany \\ ${ }^{3}$ Institute of Biomedical Engineering, Karlsruhe Institute of Technology, Karlsruhe, Germany
}

\begin{abstract}
The monodomain model and finite element method are often used together to compute electrical excitation conduction in cardiac tissue. It is known that the choice of using mass lumping as well as the used ionic current integration method affect the resulting conduction velocities $(C V s)$, especially at coarse resolutions. We describe how the regularity of node arrangement in tetrahedral grids also affects simulated CVs in a similar magnitude. We compare activation times (ATs) over a distance of $21.4 \mathrm{~mm}$ at different resolutions to a high resolution reference solution from a previously published benchmark. We show that triangulated grids are able to be within 10\% of the reference solution up to a grid resolution of $0.6 \mathrm{~mm}$, while results from regular grids already diverge by more than that at $0.4 \mathrm{~mm}$. At $0.7 \mathrm{~mm}$, a regular grid yields an AT of $80.01 \mathrm{~ms}$, where a triangulated grid with less nodes results in $47.52 \mathrm{~ms}$ (reference solution $42.82 \mathrm{~ms}$ ). We investigate how gradual perturbation of nodes from a regular grid effects $A T$, finding that $C V$ monotonically increases with degree of node perturbation.
\end{abstract}

\section{Introduction}

The mono- and bidomain models are used in computing electrical excitation propagation in cardiac tissue. For doing so, a partial differential equation is solved on a discretized domain representing the tissue, hereafter referred to as grid. Popular methods for discretizing the domain are the finite differences method (FDM) and the finite element method (FEM). While the FDM is simple to implement, discretization of a domain is generally restricted to dividing each spatial dimension with equidistant nodes. The FEM allows more flexible discretization, dividing the domain into elements of different possible shapes, resulting in more or less unstructured node distributions. The FEM is therefore especially able to discretize anatomical struc- tures with less nodes than the FDM, particularly if different regions of the domain require different resolutions. Using less nodes (and thus unstructured FEM grids) is often desirable to reduce computational effort (i.e., computation time) needed to simulate a particular problem.

A benchmark published in 2011 compared eleven simulation softwares that are using the monodomain equation with FDM or FEM [1]. It was found that of the three participating FEM solvers, two performed significantly better at lower resolutions than all FDM solvers. Of these two, one slightly overestimated conduction velocity (CV) at a low resolution, while the other one slightly underestimated it. The third FEM solver and all FDM solvers greatly underestimated $\mathrm{CV}$ in that case. The variability among FEM solvers was in a later publication attributed to the use of a consistent (vs. lumped) mass matrix, and the used ionic current integration scheme [2].

During our own studies while implementing an FEM solver, we found that the node distribution in the discrete grid also affects the resulting $\mathrm{CV}$ in a similar magnitude as the above mentioned influences. The effects are described, studied, and quantified in this work.

\section{Methods}

\subsection{The Monodomain Model}

The monodomain model is commonly stated as the partial differential equation

$$
\nabla \cdot\left(\boldsymbol{\sigma} \nabla V_{m}\right)=\beta\left(C_{m} \frac{\partial V_{m}}{\partial t}+I_{i o n}\right)
$$

with boundary condition $\boldsymbol{n} \cdot\left(\boldsymbol{\sigma} \nabla V_{m}\right)=0$ [3]. In these equations, $V_{m}$ represents the transmembrane voltage (TMV), $\sigma$ is the electrical conductivity tensor, $\beta$ the cell surface to volume ratio, and $C_{m}$ the respective cells' specific membrane capacitance per surface area. $I_{i o n}$ is the ionic current density between the intra- and extracellular 
domain. It is usually calculated using mathematical cell models that require solving a system of coupled ordinary differential equations. The boundary condition includes the outward facing normal vector $\boldsymbol{n}$ of the simulation domain's surface, therefore stating that no current leaves or enters the domain via its surface.

To compute excitation propagation using the monodomain model, the above equation has to be solved for $V_{m}$ over time, or (in practice) in a time-stepping manner. To be able to do so computationally, the spatial domain of the equation is discretized, resulting in a grid, where values of $V_{m}$ are computed for every node.

\subsection{The Finite Element Method}

The FEM allows spatially discretizing the monodomain equation by transforming it into a so-called weak formulation and constraining the solution space by reducing the degrees of freedom to the number of nodes in the grid. Ultimately, it poses linear equations only for every combination of nodes in the grid that are part of the same element.

Combining these equations for all nodes results in a linear system of equations. It can be stated in matrix-vector notation as

$$
\boldsymbol{K} \boldsymbol{v}=\beta \boldsymbol{M}\left(C_{m} \frac{\partial \boldsymbol{v}}{\partial t}+\boldsymbol{i}\right)
$$

Here, $\boldsymbol{K}$ and $\boldsymbol{M}$ are known as stiffness matrix and mass matrix, respectively. The vectors $\boldsymbol{v}$ and $\boldsymbol{i}$ contain the nodal values of the TMV and ionic current density, respectively. Resolving the partial temporal derivative using a $\theta$-algorithm results in

$$
\begin{aligned}
&\left(\frac{\beta C_{m}}{\Delta t} \boldsymbol{M}+\right.\theta \boldsymbol{K}) \boldsymbol{v}^{t+\Delta t}= \\
&\left(\frac{\beta C_{m}}{\Delta t} \boldsymbol{M}-(1-\theta) \boldsymbol{K}\right) \boldsymbol{v}^{t}-\beta \boldsymbol{M} \boldsymbol{i}^{t},
\end{aligned}
$$

where $\Delta t$ is the integration time step and superscript indices denote the specific point in time at which the variable is evaluated [3].

The results presented below were obtained solving these linear systems as an initial value problem for $\boldsymbol{v}$, using the MINRES iterative solver from the PETSc linear algebra framework [4]. Systems were preconditioned using the Eisenstat variant of the SOR method. The vector $\boldsymbol{v}$ was initialized with the resting membrane voltage of the cell model. A mathematical cell model is evaluated at each node and for every time step to populate the vector $\boldsymbol{i}$.

\subsection{Simulation Setup and Evaluation}

In order to be able to compare our results as directly as possible, we used the same simulation setups as in the 2011 benchmark publication by Niederer et al. [1]. Specifically, the simulation domain was a block of $3 \mathrm{~mm}$ by $7 \mathrm{~mm}$ by $20 \mathrm{~mm}$. A stimulus current was applied to a volume of $1.5 \mathrm{~mm}$ by $1.5 \mathrm{~mm}$ by $1.5 \mathrm{~mm}$ in one corner. The conductivity tensor had an anisotropy ratio of approx. $7.58 \mathrm{in} \mathrm{long}$ direction and was constant over the domain.

For our work, the activation time (with a threshold of $0 \mathrm{mV}$ ) of the node diagonally opposite the stimulation corner (i.e., furthest from it) was used as a surrogate measure for the CV. This node will hereafter be referred to as the distal node. The benchmark provides a reference solution for the activation time, $42.82 \mathrm{~ms}$, obtained by averaging results from three solvers at a higher resolution.

\subsection{Meshing and Perturbation}

For any spatial resolution $h$ investigated, two different grids were created for the above mentioned simulation domain. For the first set, regular FEM grids, nodes were placed equidistantly in $x$-, $y$-, and $z$-direction with $\Delta x=\Delta y=\Delta z=h$. This grid thus uses the same node positions as an FDM grid would. Each cubic cell of eight nodes was deterministically filled with six tetrahedral elements.

A second set of tetrahedral grids was created by Delaunay triangulation using the software Gmsh [5]. The target resolutions were set to $h$. It was made sure that the grids created with Gmsh had a higher average edge length than the corresponding regular grid, to ensure that better results are not the result of an accidentally better resolution.

To be able to quantify the effect of irregular node distribution gradually, additional perturbed grids have been created from the regular FEM grids. A perturbed grid was defined by its spatial resolution $h$ and perturbation factor $p$. Starting from a uniform node distribution $(\Delta x=\Delta y=$ $\Delta z=h$ ), every node was moved randomly in $x-, y-$, and $z$-direction within the interval $\left[-\frac{p h}{2}, \frac{p h}{2}\right)$ defined by a perturbation factor $p$. Nodes on the surface were only moved in a way such that the surface was not altered.

\section{Results}

\subsection{Grids}

Regular and Delaunay-triangulated grids have been created using the methods described above for each spatial resolution $h$ between $0.1 \mathrm{~mm}$ and $1 \mathrm{~mm}$ in steps of $0.1 \mathrm{~mm}$. Additionally, perturbed meshes with $p$ between 0.1 and 1 in steps of 0.1 were created. Figure 1 shows exemplary grids at a resolution of $0.5 \mathrm{~mm}$.

Figure 2 shows that a higher perturbation consistently leads to higher average edge lengths. Considering that the number of nodes and cells, and thus the average cell vol- 
A

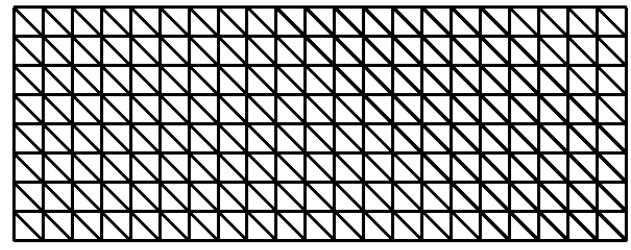

C

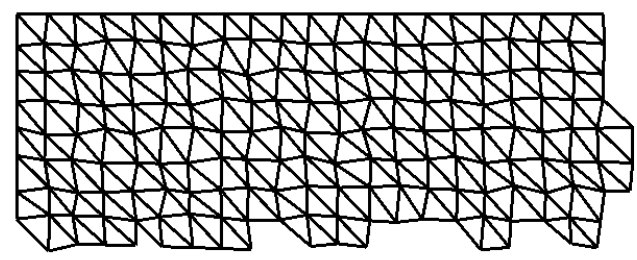

B
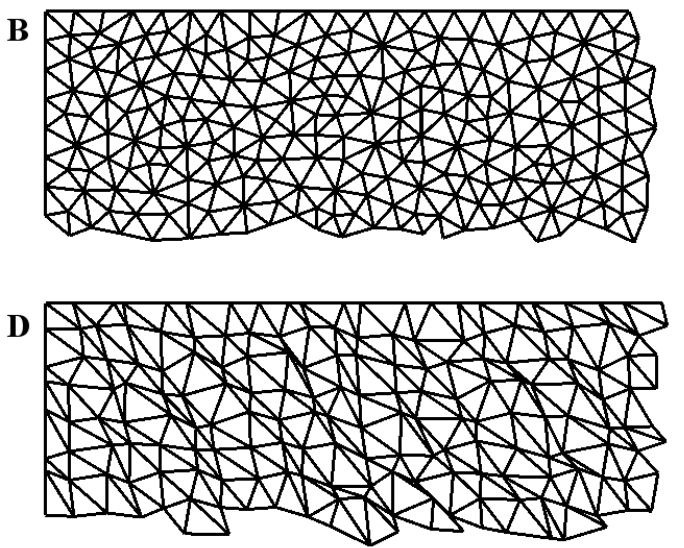

Figure 1. Upper left sections of surfaces of generated meshes at $h=0.5 \mathrm{~mm}$. A. Regular FEM grid. B. Created using Gmsh. C, D. Perturbed regular grids ( $p=0.3$ and $p=0.7$, respectively).

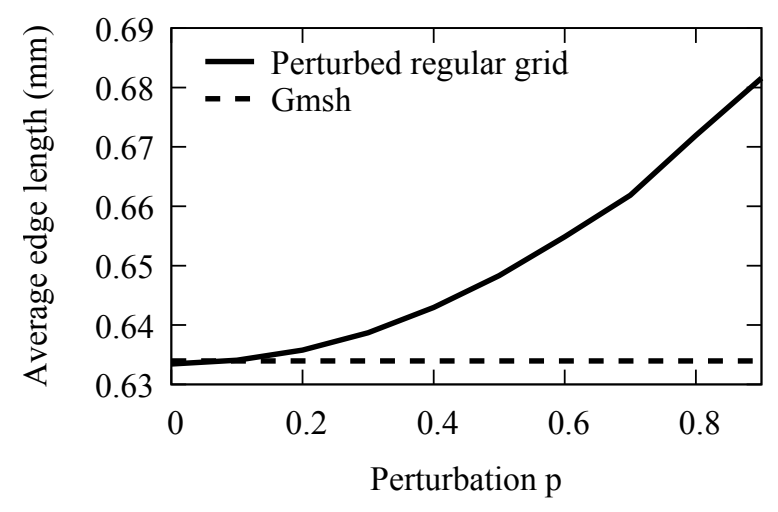

Figure 2. Average edge length of FEM grids depending on perturbation factor $p$ (solid line) at $h=0.5 \mathrm{~mm}$. Dashed line shows average edge length of corresponding Gmsh triangulated grid (no perturbation applied).

ume, does not change with perturbation, it is apparent that the mesh quality worsens with higher perturbation factors.

\subsection{Simulation Results}

Excitation conduction was simulated on the various grids according to the benchmark setup. Figure 3 shows some resulting exemplary TMV distributions. As evident in figure 4, using an unstructured grid, as resulting from Gmsh, results in a more consistent $\mathrm{CV}$ even with very coarse resolutions. In fact, up to $h=0.6 \mathrm{~mm}$, the results are within $10 \%$ of the benchmark reference solution (when also using the consistent mass matrix). Similar to the effects of using mass lumping, the effect of using a regular over a triangulated grid is stronger for more coarse resolutions.

We found that a higher perturbation factor $p$ generally
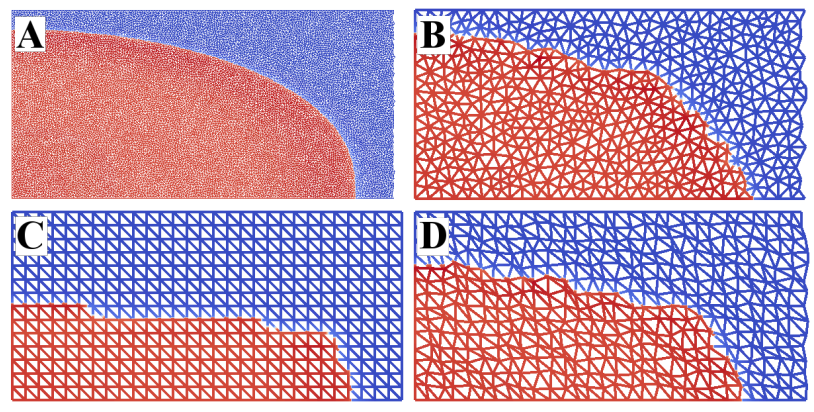

Figure 3. TMV distributions at $t=20 \mathrm{~ms}$ on the surface of different grids. Red area is activated. A. Gmsh, $\Delta x=$ $0.1 \mathrm{~mm}$. B. Gmsh, $\Delta x=0.5 \mathrm{~mm}$. C. Regular, $\Delta x=$ $0.5 \mathrm{~mm}$. D. Perturbed, $\Delta x=0.5 \mathrm{~mm}, p=0.5$.

leads to faster simulated CVs on the resulting grid. This effect is more pronounced, the more coarse a grid is. Both tendencies are illustrated exemplarily in figure 5. Notably, the results at $\Delta x=0.2 \mathrm{~mm}$ (where the $\mathrm{CV}$ is overestimated in all cases, cf. fig. 4) do not improve towards the reference solution at all by perturbation.

\section{Conclusions}

The results show that under certain conditions, very coarse grids are sufficient to reproduce an excitation pattern, provided the grid nodes are distributed sufficiently irregular. For coarse resolutions, the contribution of node regularity to simulated $\mathrm{CV}$ is of similar magnitude as the choice of using mass lumping. Figure 3 shows how the regular grid apparently imposes a prevalent direction of excitation conduction, thereby distorting the excitation propagation.

The experiments with grid perturbation show, however, that a more chaotic distribution of nodes does not necessar- 


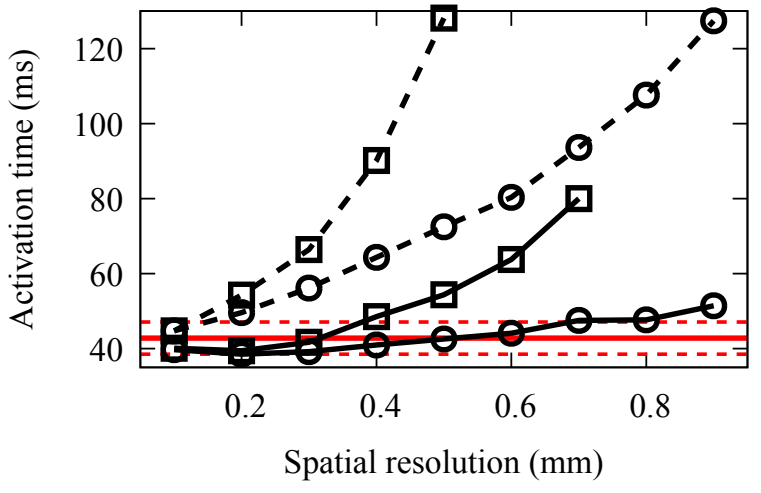

Figure 4. Activation time of distal node over grid resolutions $h$. Solid lines: Simulations with consistent mass matrix. Dashed lines: with mass lumping. Squares mark regular grid results, circles results on Gmsh grids. Red: benchmark reference solution (42.82 ms, dashed: $\pm 10 \%$ ).

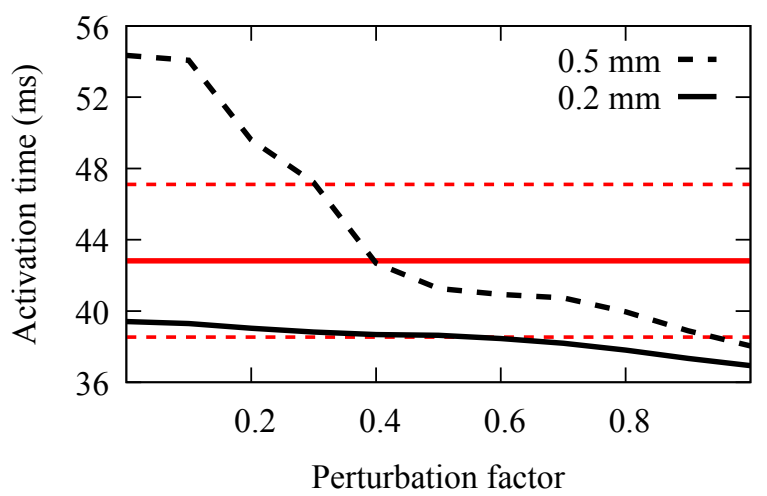

Figure 5. Distal node activation time for two resolutions over perturbation factor $p$. Red lines like in fig. 4 .

ily lead to better results. Instead, CV will just be faster for more perturbed grids, even when mesh quality worsens.

\section{Limitations and Outlook}

Our work focuses solely on the effects of grid regularity on excitation propagation in monodomain FEM simulations. It is to be expected that similar effects will occur in bidomain simulations. These effects, as well as effects on electrical field calculations (e.g., for ECG calculations) should be investigated in future works.

In this work we have only considered FEM grids with linear tetrahedral elements. It should be investigated whether similar effects occur using other geometrical elements (e.g., hexahedra), higher order elements, and other node shape functions (e.g., serendipity elements).

Our results show a correlation between $\mathrm{CV}$ and node perturbation, that is, deviation of the node placement from regular arraying. In this work, node distribution was treated the same for every spatial distribution. It is, however, to be expected that orientation of an anisotropic conductivity tensor modulates the influence of regularity in its major axis directions. Ultimately, a measure for the node regularity of an arbitrary FEM grid, possibly considering the conductivity tensor, should be established to be able to estimate the magnitude of these effects before simulations. Even accounting for expected deviations by means of a mathematical correction might be possible and desired.

\section{Acknowledgements}

The work of EMW was in parts supported by a Visiting Scholar grant of the Simula School of Research and Innovation (SSRI). He acknowledges helpful discussions with Drs. Johan Hake, Joakim Sundnes and Glenn Terje Lines of Simula Research Laboratory, Lysaker, Norway.

\section{References}

[1] Niederer SA, Kerfoot E, Benson AP, Bernabeu MO, Bernus O, Bradley C, Cherry EM, Clayton R, Fenton FH, Garny A, Heidenreich E, Land S, Maleckar M, Pathmanathan P, Plank G, Rodríguez JF, Roy I, Sachse FB, Seemann G, Skavhaug $\mathrm{O}$, Smith NP. Verification of cardiac tissue electrophysiology simulators using an N-version benchmark. Philosophical transactions Series A Mathematical physical and engineering sciences 2011;369:4331-51.

[2] Pathmanathan P, Bernabeu MO, Niederer SA, Gavaghan DJ, Kay D. Computational modelling of cardiac electrophysiology: explanation of the variability of results from different numerical solvers. International journal for numerical methods in biomedical engineering 2012;28:890-903.

[3] Sundnes J, Lines GT, Cai X, Nielsen BF, Mardal KA, Tveito A. Computing the Electrical Activity in the Heart. Berlin/Heidelberg: Springer-Verlag, 2006.

[4] Balay S, Abhyankar S, Adams MF, Brown J, Brune P, Buschelman K, Dalcin L, Eijkhout V, Gropp WD, Kaushik D, Knepley MG, McInnes LC, Rupp K, Smith BF, Zampini $\mathrm{S}$, Zhang H, Zhang H. PETSc users manual. Technical Report ANL-95/11 - Revision 3.7, Argonne National Laboratory, 2016. URL http: / /www. mcs . anl.gov/petsc.

[5] Geuzaine C, Remacle JF. Gmsh: a three-dimensional finite element mesh generator with built-in pre- and postprocessing facilities. International Journal for Numerical Methods in Engineering 2009;79:1309-31.

Address for correspondence:

Eike M. Wülfers

Institut für Experimentelle Kardiovaskuläre Medizin

Elsässer Str. 2q

79110 Freiburg

Germany

eike.wuelfers@universitaets-herzzentrum.de 